\title{
WEAK INCIDENCE ALGEBRA AND MAXIMAL RING OF QUOTIENTS
}

\author{
SURJEET SINGH and FAWZI AL-THUKAIR
}

Received 12 November 2003

\begin{abstract}
Let $X, X^{\prime}$ be two locally finite, preordered sets and let $R$ be any indecomposable commutative ring. The incidence algebra $I(X, R)$, in a sense, represents $X$, because of the wellknown result that if the rings $I(X, R)$ and $I\left(X^{\prime}, R\right)$ are isomorphic, then $X$ and $X^{\prime}$ are isomorphic. In this paper, we consider a preordered set $X$ that need not be locally finite but has the property that each of its equivalence classes of equivalent elements is finite. Define $I^{*}(X, R)$ to be the set of all those functions $f: X \times X \rightarrow R$ such that $f(x, y)=0$, whenever $x \nless y$ and the set $S_{f}$ of ordered pairs $(x, y)$ with $x<y$ and $f(x, y) \neq 0$ is finite. For any $f, g \in I^{*}(X, R), r \in R$, define $f+g, f g$, and $r f$ in $I^{*}(X, R)$ such that $(f+g)(x, y)=$ $f(x, y)+g(x, y), f g(x, y)=\sum_{x \leqslant z \leqslant y} f(x, z) g(z, y), r f(x, y)=r \cdot f(x, y)$. This makes $I^{*}(X, R)$ an $R$-algebra, called the weak incidence algebra of $X$ over $R$. In the first part of the paper it is shown that indeed $I^{*}(X, R)$ represents $X$. After this all the essential onesided ideals of $I^{*}(X, R)$ are determined and the maximal right (left) ring of quotients of $I^{*}(X, R)$ is discussed. It is shown that the results proved can give a large class of rings whose maximal right ring of quotients need not be isomorphic to its maximal left ring of quotients.
\end{abstract}

2000 Mathematics Subject Classification: 16S60, 16S90, 16W20.

1. Introduction. Let $X$ and $X^{\prime}$ be two locally finite, preordered sets, and let $R$ be a commutative ring. Under what conditions are incidence rings $I(X, R)$ and $I\left(X^{\prime}, R\right)$ isomorphic? In particular, under what conditions on $R$ can one conclude that $X$ and $X^{\prime}$ are isomorphic, when the two incidence rings $I(X, R)$ and $I\left(X^{\prime}, R\right)$ are isomorphic? The latter question has been discussed by many authors. One of the earliest results in this direction is by Stanley [9], who proved that if $R$ is a field, then the two incidence rings are isomorphic if and only if $X$ and $X^{\prime}$ are isomorphic. Froelich [4] extended this result to the case of an indecomposable ring $R$. Similar questions have been examined in $[1,3,10]$ in case $R$ need not be commutative.

Now consider any preordered set $X$ that need not be locally finite. Two elements $x, y \in X$ are said to be equivalent, $x \sim y$, if $x \leq y \leq x$. In Section 3, the isomorphism problem for weak incidence algebras is discussed. Let $X$ and $X^{\prime}$ be two preordered sets in each of which every equivalence class is finite, and let $R, R^{\prime}$ be two commutative rings such that the weak incidence algebras $I^{*}(X, R)$ and $I^{*}\left(X^{\prime}, R^{\prime}\right)$ are isomorphic as rings. In case $R$ and $R^{\prime}$ are indecomposable, Theorem 3.10 shows that $X, X^{\prime}$ are isomorphic and $R, R^{\prime}$ are isomorphic. The main aim of Section 4 is to prove some results that can help in studying the maximal ring of quotients of an $I^{*}(X, R)$. Similar work has been done in a recent paper [2] for certain classes of incidence algebras. In [7], Spiegel determines some essential ideals of an incidence algebra of a locally finite, partially 
ordered set. Here we are in a position to determine all the essential one-sided ideals of an $S=I^{*}(X, R)$ whenever $R$ is indecomposable. A particular essential right ideal $T$ is isolated and the ring $Q=\operatorname{Hom}_{S}(T, T)$ is discussed in Theorems 4.8, 4.9, and 4.10. This ring $Q$ is used to give some results on maximal right (left) ring of quotients of $S$.

2. Preliminaries. All rings considered here are with identity $1 \neq 0$. As the various concepts discussed here for weak incidence algebras are similar to those for incidence algebras, for details on incidence algebras one may consult [8]. We now collect some results on rings and modules.

LEMMA 2.1. For any commutative ring $R$ and any positive integer $n$, if $M_{R}=R^{(n)}$ is isomorphic to its summand $N$, then $M=N$.

Proof. Now $M=N \oplus K$. For any maximal ideal $P$ of $R$, the localization $M_{P}=N_{P} \oplus K_{P}$. As the ranks of the free $R_{P}$-modules $M_{P}$ and $N_{P}$ are the same and finite, $K_{P}=0$. Hence $K=0$.

LEMMA 2.2. Let $R$ be a commutative ring and let $K$ be any ring such that $M_{n}(R) \cong$ $M_{m}(K)$. Then $m$ divides $n$. If $n=m$, then $R \cong K$.

Proof. The first part follows from Wedderburn's structure theorem for simple artinian algebras, and the second part is in [6].

LEMMA 2.3. Let $T$ be any ring and let $e, e^{\prime}, f, f^{\prime}$ be any four idempotents in $T$ such that $e T \cong e^{\prime} T, f T \cong f^{\prime} T$. Then $e T f \neq 0$ if and only if $e^{\prime} T f^{\prime} \neq 0$.

Proof. The hypothesis gives that $\operatorname{Hom}_{T}(f T, e T) \cong \operatorname{Hom}_{T}\left(f^{\prime} T, e^{\prime} T\right), e T f \cong e^{\prime} T f^{\prime}$, as abelian groups. This proves the result.

3. Isomorphism. Let $X$ be any preordered set (i.e., $X$ is a set with a relation $\leqslant$ that is reflexive and transitive). For any $x, y \in X$, set $x \sim y$, if $x \leqslant y \leqslant x$. Then $\sim$ is an equivalence relation. A preordered set $X$ is said to be a class finite, preordered set if, for any $x \in X$, the equivalence class $[x]=\{y \in X: x \leq y \leq x\}$ is finite. Henceforth we take $X$ to be a class finite, preordered set and $R$ a commutative ring. The set $K^{*}(X, R)=$ $\left\{f \in I^{*}(X, R): f(x, y)=0\right.$ whenever $\left.x \sim y\right\}$ is a nil ideal. Indeed, given $f \in K^{*}(X, R)$, $f^{m+1}=0$, for $m=\left|S_{f}\right|$. Indeed, one can see that each member of $K^{*}(X, R)$ is strongly nilpotent, as defined in [8, page 176$]$, so $K^{*}(X, R)$ is contained in the lower nil radical of $I^{*}(X, R)$. Let $Y$ be a representative partially ordered subset of $X$. For any $x \in X$, let $|[x]|=n_{x}$. For each $x \in X$, the set $B_{x}=\left\{f \in I^{*}(X, R): f(u, v)=0\right.$ whenever $u+x$ or $v \nsim x$, is a ring with $\delta_{x}$ as identity, where $\delta_{x}(u, v)=0$, whenever $u \nsim x, v \nsim x$, or $u \neq v$, and $\delta_{x}(u, u)=1$ whenever $u \sim x$. Let $\delta$ denote the identity element of $I^{*}(X, R)$. For any $x, y \in X$, with $x \leqslant y$, let $e_{x y} \in I^{*}(X, R)$ be such that $e_{x y}(u, v)=0$, for $(u, v) \neq$ $(x, y)$, and $e_{x y}(x, y)=1$. Each of $e_{x y}$ is called a matrix unit of $I^{*}(X, R)$. We write $e_{x}=$ $e_{x x}$. Then $B_{x}$ is the $n_{x} \times n_{x}$ full matrix ring over $R$ with $\left\{e_{u v}: u \sim x, v \sim x\right\}$ as its set of matrix units. Let $M_{n}(R)$ denote the $n \times n$ full matrix ring over $R$. Further, $D^{*}(X, R)=$ $\left\{f \in I^{*}(X, R): f(u, v)=0\right.$ whenever $\left.u \nsim v\right\}$ is a subring of $I^{*}(X, R)$, each $B_{x}$ is an ideal of $D^{*}(X, R)$. Set $S=I^{*}(X, R), K=K^{*}(X, R), D=D^{*}(X, R)$. For any subset $Z$ of $X$, let $E_{Z} \in S$ be such that $E_{Z}(u, u)=1$ for $u \in Z$, and $E_{Z}(x, y)=0$ otherwise. For any 
$f \in S$, support of $f$, denoted by $\operatorname{suppt}(f)$, equals $\{(x, y): f(x, y) \neq 0\}$, the cardinality of suppt $(f)$ is called the weight of $f$ and we denote it by $w t(f)$. Let $X^{\prime}$ be another class finite, preordered set. Let $R^{\prime}$ be another commutative ring. We use the same symbols for the matrix units of $I^{*}(X, R)$ or $I^{*}\left(X^{\prime}, R^{\prime}\right)$ and so on, but $S^{\prime}=I^{*}\left(X^{\prime}, R^{\prime}\right), K^{\prime}=K^{*}\left(X^{\prime}, R^{\prime}\right)$, and $D^{\prime}=D^{*}\left(X^{\prime}, R^{\prime}\right)$. Let $Y$ and $Y^{\prime}$ be fixed representative partially ordered subsets of $X$ and $X^{\prime}$, respectively. For any two distinct members $y, z$ of $Y, \delta_{y}, \delta_{z}$ are orthogonal idempotents. Any $f \in S$ will be sometimes denoted by the formal sum $\sum_{x, y} f(x, y) e_{x y}$ (or by the matrix $[f(x, y)]$ indexed by $X$ ). The following is obvious.

LEMMA 3.1. (i) $I^{*}(X, R)=D^{*}(X, R) \oplus K^{*}(X, R)$ as abelian groups.

(ii) $D^{*}(X, R)=\prod_{y \in Y} B_{y}$, where $Y$ is any representative partially ordered subset of $X$.

(iii) $I^{*}(X, R) / K^{*}(X, R) \cong \Pi_{y \in Y} M_{n_{y}}(R) \cong D^{*}(X, R)$, where $Y$ is any representative partially ordered subset of $X$.

(iv) For any $f, e_{x y} \in I^{*}(X, R), w t\left(f e_{x y}\right)$ is finite, that is, $f e_{x y}=\sum_{u \leq y} a_{u y} e_{u y}$, with finitely many $a_{u x} \neq 0$.

It follows from (ii) that $K^{*}(X, R)$ does not equal the Jacobson radical of $S$, unless the Jacobson radical of $R$ is zero. For any $f \in S$, we write $f=f_{D}+f_{K}$ with $f_{D} \in D$ and $f_{K} \in K ; f_{D}$ is called the diagonal of $f$. The following is obvious.

LEMMA 3.2. For any nonempty subset $Z$ of $X, E_{Z} S E_{Z} \cong I^{*}(Z, R)$.

LEMMA 3.3. For any two idempotents $f, g \in S, f S g \neq 0$ if and only if $f_{D} S g_{D} \neq 0$.

Proof. In $\bar{S}=S / K, f+K=f_{D}+K$. As $K$ is nil, we get $f S \cong f_{D} S$. After this, Lemma 2.3 completes the proof.

LEMMA 3.4. Let $0 \neq e=e^{2} \in S$.

(i) $e_{D}$ is a nonzero idempotent and $e_{D} \delta_{y}=\delta_{y} e_{D}$ for any $y \in Y$.

(ii) There exists $y \in Y$ such that $e_{D} \delta_{y}=\delta_{y} e_{D} \neq 0$.

(iii) For any $y \in Y, e^{\prime}=e e_{D} \delta_{y} e$ is an idempotent such that $e^{\prime}(u, v)=\sum e\left(u, w_{1}\right) e\left(w_{1}\right.$, $\left.w_{2}\right) e\left(w_{2}, v\right)$, where the summation runs over $w_{1}, w_{2}$ in $[y] \cap[u, v]$. Further, $e-e^{\prime}, e^{\prime}$ are orthogonal idempotents. If $e_{D} \delta_{y} \neq 0$, then $e^{\prime} \neq 0$.

Proof. (i) is obvious. Now $S / K=\bar{D}=\Pi_{y \in K} \overline{B_{y}} \cong D, \bar{\delta}=\Pi \overline{\delta_{y}}$, and $\bar{e}=\overline{e_{D}}$. It follows that for some $y \in Y, \bar{e} \overline{\delta_{y}}=\overline{e_{D}} \overline{\delta_{y}} \neq 0$. This proves (ii). Consider any $y \in Y$ and $e^{\prime}=e e_{D} \delta_{y} e$. The definition of the product of two members of $S$ gives that $e^{\prime}(u, v)=$ $\sum e\left(u, w_{1}\right) e\left(w_{1}, w_{2}\right) e\left(w_{2}, v\right)$, where the summation runs over all $w_{1}, w_{2}$ in $[y] \cap[u, v]$. Then we have $\left(e^{\prime}\right)^{2}(u, v)=\sum_{u \leqslant w \leqslant v} e^{\prime}(u, w) e^{\prime}(w, v)=\sum e\left(u, w_{1}\right) e\left(w_{1}, w_{2}\right) e\left(w_{2}, w\right) e(w$, $\left.w_{3}\right) e\left(w_{3}, w_{4}\right) e\left(w_{4}, v\right)$, where summation runs over all $w_{i}, w$ in $[y] \cap[u, v]$ such that $w_{2} \leqslant w \leqslant w_{3}$. Thus $\left(e^{\prime}\right)^{2}(u, v)=\sum e\left(u, w_{1}\right) e\left(w_{1}, w_{4}\right) e\left(w_{4}, v\right)=e^{\prime}(u, v)$. Hence $e^{\prime}$ is an idempotent. As $e e^{\prime}=e^{\prime}=e^{\prime} e$, it follows that $e-e^{\prime}$ is an idempotent orthogonal to $e^{\prime}$. If $e_{D} \delta_{y} \neq 0$, as obviously $\overline{e^{\prime}}=\overline{e_{D} \delta_{y}}$ in $S / K$, we get $e^{\prime} \neq 0$.

LEMMA 3.5. (i) If $e \in S$ is an indecomposable idempotent, then there exists a unique $y \in Y$ such that $e=e e_{D} \delta_{y} e$.

(ii) Let $e \in S$ be a nonzero idempotent such that $e_{D} \in B_{y}$ for some $y \in Y$. Then $e=$ $e e_{D} \delta_{y} e$; this $y$ is uniquely determined by e. 
Proof. (i) In $\bar{S}=S / K, \bar{e}=\overline{e_{D}}$ is an indecomposable idempotent. So there exists a unique $y \in Y$ such that $\bar{e}=\overline{e_{D}} \overline{\delta_{y}}$. By Lemma 3.4(iii), $e^{\prime}=e e_{D} \delta_{y} e$ is a nonzero idempotent. As $e-e^{\prime}$ is orthogonal to $e^{\prime}$ and $e$ is indecomposable, $e=e^{\prime}$.

(ii) The hypothesis gives $\bar{e}=\overline{e e_{D} \delta_{y} e}$. Then Lemma 3.4(iii) gives $e=e e_{D} \delta_{y} e$.

THEOREM 3.6. Let $R$ be any indecomposable commutative ring and $X$ any class finite, preordered set. Then for any automorphism $\sigma$ of $S=I^{*}(X, R), \sigma(K)=K$.

Proof. Consider any $f \in S \backslash K$. For some $x \sim y, f(x, y) \neq 0$. Then $g=e_{x} f e_{y x}$ is such that $g(x, x) \neq 0$ and $g=e_{x} g e_{x}$. So $\sigma(g)=e \sigma(g) e$, where $e=\sigma\left(e_{x}\right)$ is an indecomposable idempotent. Let $Y$ be a representative partially ordered subset of $X$. By Lemma 3.5, there exists unique $z \in Y$ such that $e=e e_{D} \delta_{z} e, e_{D} \in B_{z}$. Thus $\sigma(g)=$ $e e_{D} \delta_{z} e \sigma(g) e e_{D} \delta_{z} e \neq 0, \delta_{z} e \sigma(g) e e_{D} \delta_{z} \neq 0$, so for some $u, v \in[z], \sigma(g)(u, v) \neq 0$. Hence $\sigma(g) \notin K$. Consequently, $\sigma(f) \notin K$. This proves the result.

LEMmA 3.7. For some $y, y^{\prime} \in Y$, let there exist idempotents $e \in B_{y}, f \in B_{y^{\prime}}$ such that $e S f \neq 0$. Then $e_{y} S e_{y^{\prime}} \neq 0$.

Proof. The hypothesis gives that $\delta_{y} S \delta_{y^{\prime}} \neq 0$, so there exist $u \in[y], v \in\left[y^{\prime}\right]$ such that $e_{u} S e_{v} \neq 0$. After this, Lemma 2.3 completes the proof.

LEMMA 3.8. If for some idempotent $f \in S, f S \cong \delta_{y} S$ for some $y \in Y$, then $f_{D}=\delta_{y}$.

Proof. We have $f_{D} S \cong \delta_{y} S$. In $\bar{S}=S / K, \overline{f_{D} S} \cong \overline{\delta_{y} S}$, so $f_{D} \in B_{y}$ and $f_{D} B_{y} \cong B_{y}$. By Lemma 2.1, $f_{D}=\delta_{y}$.

LEMMA 3.9. Let $R, R^{\prime}$ be indecomposable and $\sigma: S \rightarrow S^{\prime}$ an isomorphism.

There exists a one-to-one mapping $\eta$ of $Y$ onto $Y^{\prime}$ such that $\sigma\left(\delta_{y}\right)=\delta_{\eta(y)}+g_{\eta(y)}$ for some $g_{y} \in K^{\prime},|[y]|=|[\eta(y)]|$, and $R \cong R^{\prime}$.

Proof. The hypothesis gives that for any $x \in X, e_{x}$ is an indecomposable idempotent in $S$. Now $\sigma\left(\delta_{y}\right) S^{\prime}=\oplus \sum_{u \sim y} \sigma\left(e_{u}\right) S^{\prime}$. As these $\sigma\left(e_{u}\right) S^{\prime}$ are indecomposable and isomorphic right ideals, there exist unique $\eta(y) \in Y^{\prime}$ such that each $\sigma\left(e_{u}\right)_{D} \in B_{\delta_{\eta(y)}}^{\prime}$. Consequently, $\sigma\left(\delta_{y}\right)_{D} \in B_{\eta(y)}^{\prime}$ and $\sigma\left(\delta_{y}\right)_{D} \delta_{\eta(y)}=\delta_{\eta(y)} \sigma\left(\delta_{y}\right)_{D}$. By Lemma 3.5(ii), $\sigma\left(\delta_{y}\right)=\sigma\left(\delta_{y}\right) \sigma\left(\delta_{y}\right)_{D} \delta_{\eta(y)} \sigma\left(\delta_{y}\right)$. Similarly,

$$
\sigma^{-1}\left(\delta_{\eta(y)}\right)=\sigma^{-1}\left(\delta_{\eta(y)}\right)\left(\sigma^{-1}\left(\delta_{\eta(y)}\right)\right)_{D} \delta_{z} \sigma^{-1}\left(\delta_{\eta(y)}\right)
$$

for some $z \in Y$. So, $\delta_{\eta(y)}=\delta_{\eta(y)} \sigma\left(\left(\sigma^{-1}\left(\delta_{\eta(y)}\right)\right)_{D}\right) \sigma\left(\delta_{z}\right) \delta_{\eta(y)}$. Thus, in $\overline{S^{\prime}}=S^{\prime} / K^{\prime}$,

$$
\overline{\sigma\left(\delta_{y}\right)}=\overline{\sigma\left(\delta_{y}\right) \sigma\left(\delta_{y}\right)_{D} \delta_{\eta(y)} \sigma\left(\left(\sigma^{-1}\left(\delta_{\eta(y)}\right)\right)_{D}\right) \sigma\left(\delta_{z}\right) \delta_{\eta(y)} \sigma\left(\delta_{y}\right)}
$$

In $\overline{S^{\prime}}, \overline{\delta_{\eta(y)}}$ is a central idempotent. Thus

$$
\overline{\sigma\left(\delta_{y}\right)}=\overline{\sigma\left(\delta_{y}\right) \sigma\left(\left(\sigma^{-1}\left(\delta_{\eta(y)}\right)\right)_{D}\right) \sigma\left(\delta_{z} \delta_{y}\right) \delta_{\eta(y)}},
$$


which equals zero, if $z \neq y$. Hence $z=y$ and $\eta$ is a bijection from $Y$ onto $Y^{\prime}$. We get $\overline{\sigma\left(\delta_{y}\right)}=\overline{\delta_{\eta(y)} \sigma\left(\left(\sigma^{-1}\left(\delta_{\eta(y)}\right)\right)_{D} \delta_{y}\right)}$ and $\overline{\delta_{\eta(y)}}=\overline{\delta_{\eta(y)}} \overline{\sigma\left(\left(\sigma^{-1}\left(\delta_{\eta(y)}\right)\right)_{D} \delta_{y}\right)}$. Hence $\overline{\sigma\left(\delta_{y}\right)}=\overline{\delta_{\eta(y)}}$. This shows that $\sigma\left(\delta_{y}\right)=\delta_{\eta(y)}+g_{\eta(y)}$ for some $g_{\eta(y)} \in K^{\prime}$. Now $\delta_{y} S \delta_{y}=$ $B_{y}$. As $\sigma\left(\delta_{y}\right) S^{\prime} \cong \delta_{\eta(y)} S^{\prime}$, it follows that $B_{y} \cong B_{\eta(y)}^{\prime}$. By Lemma 2.2, $|[y]|=|[\eta(y)]|$ and $R \cong R^{\prime}$.

THEOREM 3.10. Let $X$ and $X^{\prime}$ be two class finite, preordered sets. Let $R$ and $R^{\prime}$ be any two indecomposable commutative rings. If there exists an isomorphism of $I^{*}(X, R)$ onto $I^{*}\left(X^{\prime}, R^{\prime}\right)$, then $X, X^{\prime}$ are isomorphic and the rings $R, R^{\prime}$ are isomorphic.

Proof. We use the terminology developed before Theorem 3.10. Consider any $u$, $v \in Y$ such that $u \leqslant v$. Then $e_{u} S e_{v} \neq 0, \sigma\left(e_{u}\right) S^{\prime} \sigma\left(e_{v}\right) \neq 0$. It follows from Lemma 3.9 that $\sigma\left(e_{u}\right)_{D} \in B_{\eta(u)}^{\prime}, \sigma\left(e_{v}\right)_{D} \in B_{\eta(v)}^{\prime}$. By Lemma 3.3, $\sigma\left(e_{u}\right)_{D} S^{\prime} \sigma\left(e_{v}\right)_{D} \neq 0, e_{\eta(u)} S^{\prime} e_{\eta(v)} \neq$ 0 , hence $\eta(u) \leqslant \eta(v)$. Thus $\eta$ is an isomorphism of $Y$ onto $Y^{\prime}$. Also by Lemma 3.9, $|[y]|=|[\eta(y)]|$, hence it follows that $X$ and $X^{\prime}$ are isomorphic. By Lemma 3.9, $R$ and $R^{\prime}$ are isomorphic.

LEMMA 3.11. For any commutative ring $T$ and any class finite, preordered set $X$, the following hold.

(i) A central idempotent $e \in I^{*}(X, T)$ is centrally indecomposable if and only if $e=$ $g E_{Z}$ for some indecomposable idempotent $g \in T$ and a connected component $Z$ of $X$.

(ii) Let $g$ and $h$ be two indecomposable idempotents in $T$ and let $Z, Z^{\prime}$ be two connected components of $X$; the rings $g E_{Z} I^{*}(X, T), h E_{Z} I^{*}(X, T)$ are isomorphic if and only if the rings $g T, h T$ are isomorphic and $Z, Z^{\prime}$ are isomorphic.

Proof. (i) Consider any central idempotent $e \in I^{*}(X, T)$. On the same lines as for incidence algebras, it can be easily seen that $e(x, y)=0$, whenever $x \neq y$. For any connected component $Z$ of $X$, there exists an idempotent $g_{Z} \in T$ such that $e(x, x)=g_{Z}$ for every $x \in X$. Using this, (i) follows. (ii) As $g E_{Z} I^{*}(X, T) \cong I^{*}(Z, g T)$ and $h E_{Z^{\prime}} I^{*}(X, T) \cong$ $I^{*}\left(Z^{\prime} \cdot h T\right)$, the result follows from Theorem 3.10.

Let $T$ be any ring. Let $\operatorname{In}(T)$ be the set of all centrally indecomposable central idempotents of $T$. Two central idempotents $g, h$ of $T$ are said to be equivalent if the rings $g T$ and $h T$ are isomorphic. For any central idempotent $g \in T$, $[g]$ denotes the set of central idempotents in $T$ equivalent to $g$.

THEOREM 3.12. Let $R$ and $R^{\prime}$ be any two commutative rings and let $X, X^{\prime}$ be two class finite, preordered sets. Let $\sigma: I^{*}(X, R) \rightarrow I^{*}\left(X^{\prime}, R^{\prime}\right)$ be a ring isomorphism. Let $g \in \operatorname{In}(R)$ and let $Z$ be a connected component of $X$.

(i) There exist unique $g^{\prime} \in \operatorname{In}\left(R^{\prime}\right)$ and unique connected component $Z^{\prime}$ of $X^{\prime}$ such that $\sigma\left(g E_{Z}\right)=g^{\prime} E_{Z^{\prime}}$; further, $Z \cong Z^{\prime},|[g]||[Z]|=\left|\left[g E_{Z}\right]\right|=\left|\left[g^{\prime} E_{Z^{\prime}}\right]\right|=\left|\left[g^{\prime}\right]\right|\left|\left[Z^{\prime}\right]\right|$.

(ii) If the cardinalities of $[g]$ and $\left[g^{\prime}\right]$ are finite and equal, then $X$ and $X^{\prime}$ are isomorphic.

Proof. (i) The first part follows from Lemma 3.11(i); the second part follows from Lemma 3.11(ii). (ii) If $|[g]|=\left|\left[g^{\prime}\right]\right|$ and they are finite, if follows from (i) that, given any 
connected component $Z$ of $X$, there exists a connected component $Z^{\prime}$ of $X^{\prime}$ isomorphic to $Z$, and [ $Z]$, [ $\left.Z^{\prime}\right]$ have the same cardinalities. Consequently, $X$ and $X^{\prime}$ are isomorphic.

The following is immediate from Theorem 3.12.

COROLLARY 3.13. Let $R$ be any commutative ring such that $R$ admits an indecomposable idempotent $g$ for which the equivalence class $[g]$ is finite. Let $X$ and $X^{\prime}$ be any two class finite, preordered sets. If the rings $I^{*}(X, R)$ and $I^{*}(Y, R)$ are isomorphic, then $X$ and $X^{\prime}$ are isomorphic.

4. Essential right ideals and maximal ring of quotients. Throughout $S=I^{*}(X, R)$, where $X$ is a class finite, preordered set and $R$ is a commutative ring in which 1 is indecomposable. Any $x \in X$ is said to be a maximal element if the equivalence class $[x]$ is maximal in the partially ordered set of the equivalence classes in $X$. For any $x, y \in X$, we say $x<y$, if $x \leq y$ but $[x] \neq[y]$. Set $X_{0}=\{x \in X: x$ is maximal $\}$, $Y_{0}=\left\{(x, y) \in X \times X_{0}: x \leq y\right\}, Y_{1}=\{(x, y): x<y$ and there does not exist any $z \in$ $X_{0}$ such that $\left.y \leq z\right\}, Y_{2}=\left\{(x, y): x<y\right.$ and there exists a $z \in X_{0}$ such that $\left.y<z\right\}$, and $Y_{3}=\left\{(x, y) \in X_{0} \times X_{0}:[x]=[y]\right\}$. Further, $K=K^{*}(X, R)$. Now $L=\sum(x, y) \in Y_{3} e_{x y} R$ is a right ideal of $S$. In [2], maximal rings of quotients of certain incidence algebras have been discussed. Here we intend to prove some results that can help in studying the maximal rings of quotients of $S$. Spiegel [7] has determined certain classes of essential ideals of an incidence algebra of a locally finite, preordered set. Here we determine all essential one-sided ideals of $S$. For the definitions of an essential submodule, dense submodule, and singular submodule of a module, one may refer to [5]. Let $M$ be any module, then $N \subset_{e} M\left(N \subset_{d} M\right)$ denotes that $N$ is an essential (dense) submodule of $M$, and $Z(M)$ denotes the singular submodule of $M$. The concept of the maximal right ring of quotients of a ring is discussed in [5, Section 13].

LEMmA 4.1. Let $K_{1}=K+L$. Then $K_{1}$ is an essential right ideal of $S$ and $l \cdot \operatorname{ann}\left(K_{1}\right)=0$. Indeed for any $0 \neq f \in S$, there exists $e_{x w} \in K_{1}$ such that $0 \neq f e_{x w} \in K_{1}$.

Proof. Let $0 \neq f \in S$. Then $f(u, v) \neq 0$ for some $u \leq v$. Suppose $f K_{1}=0$. If $v$ is not maximal in $X$, there exists $e_{v z} \in K$, and $f e_{v z} \neq 0$, which is a contradiction. Hence $v$ is maximal. Then $e_{v} \in K_{1}$ with $f e_{v} \neq 0$, which is again a contradiction. Hence $l \cdot \operatorname{ann}\left(K_{1}\right)=$ 0 . In any case there exists $e_{x y} \in K_{1}$ such that $f e_{x y} \neq 0$. By applying induction on $w t\left(f e_{x y}\right)$, we prove that for some $g \in S, 0 \neq f e_{x y} g \in K_{1}$, which will prove that $K_{1} \subset_{e} S_{S}$. Suppose $w t\left(f e_{x y}\right)=1$. Then $f e_{x y}=a e_{u y}$, for some $0 \neq a \in R$. If $y$ is not maximal, for any $z>y, f e_{x y} e_{y z}=a e_{u z} \in K_{1}$. If $y$ is maximal, then $e_{y} \in K_{1}$, so $f e_{x y} e_{y}=a e_{u y} \in K_{1}$. To apply induction, suppose that $w t\left(f e_{x y}\right)=n>1$, and for any $h \in S$, if for some $e_{u v} \in K_{1}, w t\left(h e_{u v}\right)<n$ and $h e_{u v} \neq 0$, then for some $e_{v z} \in S, 0 \neq h e_{u v} e_{v z} \in K_{1}$. We can write $f e_{x y}=a e_{u y}+h$, where $w t(h)=n-1$ and $h(u, y)=0$. For some $e_{y s} \in K_{1}$, $a e_{u y} e_{y s}=a e_{u s} \in K_{1}$. Then $f e_{x s}=a e_{u s}+h e_{y s}$ with $w t\left(h e_{y s}\right)=n-1$. By the induction hypothesis, there exists $e_{s w} \in K_{1}$ such that $0 \neq h e_{y s} e_{s w} \in K_{1}$. Then $0 \neq f e_{x w} \in K_{1}$. Hence $K_{1} \subset_{e} S_{S}$. 
We call a subset $B$ of $R$ an essential subset of $R$ if, for each $0 \neq r \in R$, there exists an $s \in R$ such that $0 \neq r s \in B$. Clearly the ideal of $R$ generated by an essential subset is an essential ideal.

LEMmA 4.2. Let $E \subset_{e} S_{S}$. For any $x \leq y$ in $X$, let $A_{x y}=\left\{r \in R: r e_{x y} \in E\right\}, B_{x y}=$ $\cup_{y \leq z} A_{x z}$.

(i) $A_{x y} \subseteq A_{x w}$ whenever $x \leq y \leq w$.

(ii) $B_{x y}$ is an essential subset of $R$.

Proof. (i) is trivial. Let $0 \neq r \in R$. Then for some $g \in S, 0 \neq r e_{x y} g \in E$. For some $y \leq w, r g(y, w) \neq 0$. This gives $r e_{x y} g e_{w}=r g(y, w) e_{x w} \in E, r g(y, w) \in B_{x y}$. This proves that $B$ is an essential subset of $R$.

LEMMA 4.3. Let $\left\{A_{x y}\right.$ : either $x<y$, or $x \leq y$ and $y$ is maximal in $\left.X\right\}$ be a family of ideals in $R$ such that (i) $A_{x y} \subseteq A_{x z}$ whenever $y \leq z$, and (ii) for any $x \leq y$ in $X$, $B_{x y}=\cup_{y \leq z} A_{x z}$ is an essential subset of $R$. Then $E=\sum_{x, y} A_{x y} e_{x y}$ is an essential right ideal of $S$ and $E \subseteq K_{1}$.

Proof. It is easy to verify that $E$ is a right ideal of $S$ contained in $K_{1}$. Let $0 \neq f \in K_{1}$. By induction on $w t(f)$, we prove that $0 \neq f r e_{x y} \in E$ for some $e_{x y} \in K_{1}, r \in R$, which will prove that $E \subset_{e} S_{S}$. Suppose $f=a e_{x y}$. As $a \neq 0$, there exists a $z \geq y$ and an $r \in R$ such that $0 \neq a r \in A_{x z}$. Then $0 \neq f r e_{y z}=a r e_{x z} \in E$. Here, if $y$ is not maximal, choose $z>y$; if $y$ is maximal, choose $y=z$; in any case $e_{x z} \in K_{1}$. Thus the result holds for $w t(f)=1$. To apply induction, let $w t(f)=n>1$, and let the result hold for any positive integer less than $n$. We write $f=a e_{x y}+h$, with $0 \neq a \in R, e_{x y} \in K_{1}, w t(h)=n-1$, and $h(x, y)=0$. There exists an $r e_{y z} \in K_{1}$ such that $0 \neq a e_{x y} r e_{y z}=a r e_{x z} \in E$. Then $0 \neq f r e_{x z}=a r e_{x z}+h r e_{y z}$. If $h r e_{y z}=0, f r e_{x z}=a r e_{x z} \in E$ and we finish. Suppose $h r e_{y z} \neq 0$. By the induction hypothesis, there exists $b e_{z w} \in K_{1}$, with $b \in R$, such that $0 \neq$ hre $_{y z} b e_{z w} \in E$. Then $0 \neq f r b e_{x w} \in E$.

Let Minness $(S)$ be the set of all essential right ideals of the form given in Lemma 4.3.

LEMMA 4.4. $Z(S)=\{f \in S: f E=0$ for some $E \in \operatorname{Minness}(S)\}$.

Proof. Let $f \in Z(S)$. For some $E \subset_{e} S_{S}, f E=0$. By Lemmas 4.2 and 4.3, there exists an $E^{\prime} \in \operatorname{Minness}(S)$ such that $E^{\prime} \subseteq E$. Then $f E^{\prime}=0$. This proves the result.

THEOREM 4.5. $Z\left(S_{S}\right)=0$ if and only if $Z(R)=0$.

Proof. Let $Z(R) \neq 0$. For some $r \neq 0$ and an essential ideal $A$ of $R, r A=0$. In Lemma 4.3, by taking every $A_{x y}=A$, we get an $E \subset_{e} S_{S}$ such that $r I E=0$. Thus $Z(S) \neq 0$. Conversely, let $Z(S) \neq 0$. Consider any $0 \neq f \in Z(S)$. For some $E \in \operatorname{Minness}(S), f E=0$. Now $f(u, v) \neq 0$ for some $u \leq v$. Then $0 \neq e_{u} f \in Z(S)$. Suppose there exists a maximal $z \geq v$. As $z$ is maximal, it follows from Lemma 4.3(i) that $B_{v z}=A_{v z}$, so $e_{v} f e_{v z} A_{v z}=0$, $f(u, v) A_{v z}=0, f(u, v) \in Z(R)$. Hence $Z(R) \neq 0$.

Proposition 4.6. For any $(x, y) \in Y_{0}$, set $A_{x y}=R$, for $(x, y) \in Y_{1}$, set $A_{x y}=R$, and for $(x, y) \in Y_{2}$, set $A_{x y}=0$. Let $T=\sum_{x, y} e_{x y} A_{x y}$.

(i) Then $T$ is an ideal of $S, T \subset_{e} S_{S}$, and $l \cdot \operatorname{ann}(T)=0$.

(ii) $S$ embeds in the ring $Q=\operatorname{Hom}\left(T_{S}, T_{S}\right)$ such that $S_{S}$ is dense in $Q_{S}$. 
Proof. That $T$ is an essential right ideal in $S$ follows from Lemma 4.3. Suppose that $0 \neq f \in l \cdot \operatorname{ann}(T)$. Then $f(u, v) \neq 0$ for some $u \leq v$. Suppose there exists no maximal $z \geq v$. Choose any $w>v$. Then $e_{v w} \in T$ but $f e_{v w} \neq 0$, which is a contradiction. Hence there exists a maximal $z \geq v$. Then $e_{v z} \in T$ and $f e_{v z} \neq 0$, which is also a contradiction. Hence $l \cdot \operatorname{ann}(T)=0$. Consider any $e_{x y} \in T$. By Lemma 3.1, $w t\left(f e_{x y}\right)$ is finite, so $f e_{x y}=$ $\sum_{u \leq y} a_{u y} e_{u y}$, a finite sum. By definition, the following two cases arise.

CASE 1. $y$ is maximal. Then every $e_{u y} \in T$, so $f e_{x y} \in T$.

CASE 2. There does not exist any maximal $z \geq y$. Then $u<y, A_{u y}=R, e_{u y} \in T$, hence $f e_{x y} \in T$.

This proves that $T$ is an ideal in $S$. For each $f \in S$, let $\lambda(f)$ be the left multiplication on $T$ by $f$. Then $\lambda$ is an embedding of $S$ in $Q$. Consider any $\sigma, \eta \in Q$, with $\sigma \neq 0$. Then for some $f \in T, \sigma(f) \neq 0$. We see that $\sigma \cdot \lambda(f)=\lambda(\sigma(f)) \neq 0$ and $\eta \cdot \lambda(f)=\lambda(\eta(f)) \in$ $\lambda(S)$. Hence $S_{S}$ is dense in $Q_{S}$.

For each $x_{0} \in X_{0}$, set $T_{\left[x_{0}\right]}=\sum\left\{e_{x y} R:(x, y) \in Y_{3}\right.$ and $\left.\left[x_{0}\right]=[y]\right\}$, and set $T^{\prime}=$ $\sum\left\{e_{x y} R:(x, y) \in Y_{1}\right\}$. Observe that $T_{\left[x_{0}\right]}=T_{\left[x_{1}\right]}$ if and only if $\left[x_{0}\right]=\left[x_{1}\right]$. Each of $T_{\left[x_{0}\right]}, T^{\prime}$ is a right ideal of $S$ contained in $T$, and $T$ is a direct sum of these right ideals. Let $Z_{0}$ be the set of equivalence classes in $X$ given by the members of $X_{0}$. For any ring $P$, let $\hat{P}$ be the maximal right ring of quotients of $P$ [5, Section 13]. The following result can be easily deduced from various results and exercises given in [5, Sections 8 and 13].

THEOREM 4.7. (I) For any family of rings $\left\{P_{\alpha}: \alpha \in \Lambda\right\}, P=\prod_{\alpha \in \Lambda} P_{\alpha}, \widehat{P}=\prod_{\alpha \in \Lambda} \widehat{P_{\alpha}}$.

(II) For any two subrings $A, B$ of a ring $P$, if $A_{A} \subset_{d} B_{A}, B_{B} \subset_{d} P_{B}$, then $\hat{A}=\hat{P}$.

(III) For any positive integer $n$ and any ring $P, \widehat{M_{n}(P)}=M_{n}(\hat{P})$.

THEOREM 4.8. (i) $Q=\operatorname{Hom}\left(T_{S}, T_{S}\right) \cong\left(\Pi\left\{\operatorname{Hom}_{S}\left(T_{\left[x_{0}\right]}, T_{\left[x_{0}\right]}\right):\left[x_{0}\right] \in Z_{0}\right\}\right) \times \operatorname{Hom}_{S}\left(T^{\prime}\right.$, $\left.T^{\prime}\right)$.

(ii) Maximal right rings of quotients of $S$ and $Q$ are the same.

(iii) Let $P_{\left[x_{0}\right]}=\operatorname{Hom}_{S}\left(T_{\left[x_{0}\right]}, T_{\left[x_{0}\right]}\right)$ and $P^{\prime}=\operatorname{Hom}\left(T^{\prime}, T^{\prime}\right)$. Then $\widehat{S} \cong\left(\Pi\left\{\widehat{P_{\left[x_{0}\right]}}:\left[x_{0}\right] \in\right.\right.$ $\left.\left.Z_{0}\right\}\right) \times \widehat{P}^{\prime}$.

Proof. To prove (i) it is enough to prove that $\operatorname{Hom}_{S}\left(T_{\left[x_{0}\right]}, T_{\left[x_{1}\right]}\right)=0$ whenever $\left[x_{0}\right] \neq$ $\left[x_{1}\right], \operatorname{Hom}_{S}\left(T_{\left[x_{0}\right]}, T^{\prime}\right)=0=\operatorname{Hom}_{S}\left(T^{\prime}, T_{\left[x_{0}\right]}\right)$. Consider $\sigma \in \operatorname{Hom}_{S}\left(T_{\left[x_{0}\right]}, T_{\left[x_{1}\right]}\right)$. For any $e_{x y} \in T_{\left[x_{0}\right]},\left[x_{0}\right]=[y]$, so $e_{u y} \notin T_{\left[x_{1}\right]}$, but $\sigma\left(e_{x y}\right)=\sum_{u \leq y} a_{u y} e_{u y}, a_{u y} \in R$. Thus $\sigma\left(e_{x y}\right)=0, \sigma=0$. Similarly, we can prove that the others are also zero. As $S_{S}$ is dense in $Q_{S}, \widehat{S}=\widehat{Q}$. Because of (i) and Theorem 4.7, we get $\widehat{S} \cong\left(\Pi\left\{\widehat{P_{\left[x_{0}\right]}}:\left[x_{0}\right] \in Z_{0}\right\}\right) \times \widehat{P^{\prime}}$.

We now discuss matrix representations of $\operatorname{Hom}_{S}\left(T_{\left[x_{0}\right]}, T_{\left[x_{0}\right]}\right)$ and $\operatorname{Hom}_{\mathcal{S}}\left(T^{\prime}, T^{\prime}\right)$.

THEOREM 4.9. Let $x_{0}$ be a maximal member of $X, U_{x_{0}}=\left\{x \in X: x \leq x_{0}\right\}$. Then $\operatorname{Hom}_{S}\left(T_{\left[x_{0}\right]}, T_{\left[x_{0}\right]}\right)$ is isomorphic to the ring of column-finite matrices over $R$ indexed by $U_{x_{0}}$.

Proof. Let $\sigma \in \operatorname{Hom}_{S}\left(T_{\left[x_{0}\right]}, T_{\left[x_{0}\right]}\right)$. For $e_{x y} \in T_{\left[x_{0}\right]}, y \sim x_{0}$. If $\sigma\left(e_{x y}\right)=\sum_{u \leq y} a_{u y} e_{u y}$, then for any other $e_{x z} \in T_{\left[x_{0}\right]}, \sigma\left(e_{x z}\right)=\sum_{u \leq z} a_{u z} e_{u z}=\sigma\left(e_{x y}\right) e_{y z}=\sum_{u \leq y} a_{u y} e_{u z}$, $a_{u y}=a_{u z}$. Conversely, any $\sigma \in \operatorname{Hom}_{R}\left(T_{\left[x_{0}\right]}, T_{\left[x_{0}\right]}\right)$, such that if $\sigma\left(e_{x y}\right)=\sum_{u \leq y} a_{u y} e_{u y}$, 
then $\sigma\left(e_{x z}\right)=\sum_{u \leq y} a_{u y} e_{u z}$ for $y \sim z$, is in $\operatorname{Hom}_{S}\left(T_{\left[x_{0}\right]}, T_{\left[x_{0}\right]}\right)$. Now $V_{x_{0}}=\left\{e_{x y}: x \in\right.$ $\left.U_{x_{0}}, y \sim x_{0}\right\}$ is an $R$-basis of $T_{\left[x_{0}\right]}$. We write $\sigma\left(e_{x y}\right)=\sum_{u, v} a_{u v x y} e_{u v}, e_{u v} \in V_{x_{0}}$. Then $a_{u v x y}=0$, for $v \neq y, a_{u y x y}=a_{u z x z}$, whenever $y \sim z$. We write $b_{u x}=a_{u y x y}$ and $b_{u x}=0$ otherwise. We get matrix $\left[b_{u x}\right]$ over $R$ indexed by $U_{x_{0}}$. This matrix is column finite; $\sigma \leftrightarrow\left[b_{u x}\right]$ gives the desired isomorphism.

THEOREM 4.10. Let $X^{\prime}=\{y \in X$ : there exist no maximal $z \geq y\}$. Let $G$ be the set of arrays $\left[a_{v x y}\right]$ over $R$ indexed by $X^{\prime} \times X^{\prime} \times X^{\prime}$ such that it has following properties:

(i) $a_{v x y}=0$, whenever $x \nless y$, $v \nless y$, or $x<v<y$,

(ii) for any fixed pair $(x, y)$ with $x<y$, the number of $v$ for which $a_{v x y} \neq 0$ is finite,

(iii) for $y \leq z, a_{v x y}=a_{v x z}$ if $v<y$, and $a_{v x z}=0$ if $v \nless y$ and $v<z$.

In $G$, define addition componentwise and the product by $\left[a_{v x y}\right]\left[b_{v x y}\right]=\left[c_{v x y}\right]$ such that $c_{v x y}=\sum_{w} a_{v w y} b_{w x y}$. Then $\operatorname{Hom}_{S}\left(T^{\prime}, T^{\prime}\right) \cong G$.

In case $X^{\prime}$ has the property that for every pair of elements $u, v$ in $X^{\prime}$ there exists a $w \in X^{\prime}$ such that $u \leq w, v \leq w$, then any array $\left[a_{v x y}\right] \in G$ has the following additional properties:

(iv) if $u, v \in X^{\prime}$ are not comparable, then $a_{u x v}=0$,

(v) for $x<y, x<z, a_{v x y}=a_{v x z}$.

Put $b_{v x}=a_{v x y}$. Then $\left[b_{v x}\right]$ is a column finite matrix indexed by $X^{\prime}$ with the property that $b_{v x}=0$ if $v>x$, or there exists $y>x$ such that $v \nless y$. Set $b_{v x}=0$ in all other cases. Let $B$ be the set of all such matrices. Then $B$ is a ring isomorphic to $\operatorname{Hom}_{S}\left(T^{\prime}, T^{\prime}\right)$.

Proof. Let $\sigma \in \operatorname{Hom}_{S}\left(T^{\prime}, T^{\prime}\right)$. For any $x<y \leq z \in X^{\prime}$, we have $\sigma\left(e_{x y}\right)=$ $\sum c_{u v x y} e_{u v}, c_{u v x y} \in R,(u, v) \in Y_{1}$, with $c_{u v x y}=0$, whenever $v \neq y$. So we can write $\sigma\left(e_{x y}\right)=\sum_{v<y} e_{v y} a_{v x y}$, a finite sum. For $y \leq z, \sigma\left(e_{x z}\right)=\sigma\left(e_{x y}\right) e_{y z}$ gives $a_{v x y}=a_{v x z}$ for $v<y$ and $a_{v x z}=0$ whenever $v \nless y, v<z$. Suppose we have some $x<v<y$, by considering $\sigma\left(e_{x y}\right)=\sigma\left(e_{x v}\right) e_{v y}$ it follows that $a_{v x y}=0$. For any other $(v, x, y) \in$ $X^{\prime} \times X^{\prime} \times X^{\prime}$, set $a_{v x y}=0$. We get an array $\left[a_{v x y}\right]$ with the desired properties. Conversely, any such array gives an $S$-endomorphism of $T^{\prime}$. This gives the desired isomorphism.

Suppose every pair of elements in $X^{\prime}$ have a common upper bound. Consider any $v, w \in X^{\prime}$ that are not comparable. By (i), $a_{v x w}=0$ for any $x$; this proves (iv). Suppose $x<y, x<z$. There exists $w \in X^{\prime}$ such that $y<w, z<w$. Then $\sigma\left(e_{x z}\right) e_{z w}=$ $\sigma\left(e_{x y}\right) e_{y w}=\sigma\left(e_{x w}\right)$ gives (v). Set $b_{v x}=a_{v x y}$. Because of (v), $b_{v x}$ is well defined. It gives a matrix $\left[b_{v x}\right]$ indexed by $X^{\prime}$, which is column finite and has the property that $b_{v x}=0$ if either $v>x$, or there exists $y>x$ such that $v \nless y$. Let $B$ be the set of all column-finite matrices $\left[b_{v x}\right.$ ] over $R$ indexed by $X^{\prime} \times X^{\prime}$ with $b_{v x}=0$, whenever either $v>x$ or there exists a $y>x$ such that $v \nless y$. Then $\operatorname{Hom}_{S}\left(T^{\prime}, T^{\prime}\right)$ is isomorphic to the $\operatorname{ring} B$.

REMARK 4.11. Let $X$ be any locally finite, preordered set and let $R$ be any indecomposable commutative ring. Obviously, $S=I^{*}(X, R)$ is a subring of $S^{\prime}=I(X, R)$. But $S_{S}$ need not be dense or essential in $S_{S}^{\prime}$. So the maximal right rings of quotients of $S$ and $S^{\prime}$ need not be the same; in fact, they need not be isomorphic (see the example given below). In case $S_{S}$ is dense in $S^{\prime}$, the two rings will have the same maximal right ring of quotients. In that case, $S$ can help in studying $S^{\prime}$. 
THEOREM 4.12 [2]. Let $X$ be any partially ordered set such that for any $x \in X$, there exists a maximal element $z \geq x$ and $L_{z}=\{y \in X: y \leq z\}$ is finite. Let $X_{0}$ be the set of maximal elements of $X$. For each $z \in X_{0}$, let $n_{z}$ be the number of elements $y \leq z$. For the ring $S=I(X, R), \hat{S} \cong \Pi\left\{M_{n_{z}}(\hat{R})\right.$ : where $z$ runs over representatives of equivalence classes in $\left.X_{0}\right\}$.

Proof. Let $f, g \in S^{\prime}=I(X, R)$ with $g \neq 0$. For some $u, v \in X, g(u, v) \neq 0$. Then $g e_{v} \neq 0$. At the same time the hypothesis on $X$ gives that the support of $f e_{v}$ is finite, so $f e_{v} \in S=I(X, R)$. Hence $S_{S}$ is dense in $S^{\prime}$. After this, Theorems 4.7, 4.8, and 4.9 complete the proof.

EXAMPLE 4.13. Let $X=\mathbb{N}$ be the set of natural numbers and let $R$ be any indecomposable commutative ring. Consider $S=I^{*}(\mathbb{N}, R)$ and $S^{\prime}=I(\mathbb{N}, R)$. Let $0 \neq f \in S^{\prime}$. For some $r \in \mathbb{N}, f e_{r} \neq 0$. Clearly, the support of $f e_{r}$ is finite. Hence $S_{S}$ is dense in $S^{\prime}$. So the maximal right quotient rings of $S$ and $S^{\prime}$ are the same. Consider $g \in S^{\prime}$ for which $g(1, n)=1$ for every $n$, and $g(n, m)=0$ otherwise. Then for any $h \in S, h g=0$ or $h g=k g$ for some $0 \neq k \in \mathbb{N}$, so ${ }_{S} S$ is not dense in $S^{\prime}$. Thus maximal left rings of quotients of $S$ and $S^{\prime}$ are not the same. We now show that they need not be isomorphic. Consider $R=F$ a countable field. As $\mathbb{N}$ has no maximal element, $K=T=T^{\prime}, Q=\operatorname{Hom}_{S}\left(T^{\prime}, T^{\prime}\right)$. By Theorem 4.10, $Q$ is isomorphic to $S^{\prime}$. But $S^{\prime}$, as a right $S^{\prime}$-module, is dense in the ring $L$ of all column-finite matrices over $F$, indexed by $\mathbb{N}$. It is well known that the ring of all column-finite matrices over a field, indexed by any set, is right self-injective. Hence $L$ is the maximal right ring of quotients of $S$ and $S^{\prime}$. Let $\mathbb{N}^{\prime}$ be the set of natural numbers with reverse ordering. As $\mathbb{N}^{\prime}$ has unique maximal element $0, \mathbb{N}^{\prime}=T_{0}$, by Theorem 4.9 , the corresponding $Q^{\prime}$ is isomorphic to the ring of all column-finite matrices over $F$, indexed by $\mathbb{N}$. So $Q^{\prime}$ is right self-injective. However $S=I^{*}(\mathbb{N}, F)$ is anti-isomorphic to $S_{1}=I^{*}\left(\mathbb{N}^{\prime}, F\right)$. So $Q^{\prime \prime}$, the maximal left ring of quotients of $S$, is isomorphic to the ring of all row-finite matrices over $F$, indexed by $\mathbb{N}$. Now $e_{00} Q^{\prime \prime}$ is a countable set, and any minimal left ideal of $Q^{\prime \prime}$ is generated by an element of $e_{00} Q^{\prime \prime}$, so the left socle of $e_{00} Q^{\prime \prime}$ is of countable rank. For $S^{\prime}$, the left socle is $e_{00} S^{\prime}$, which is of uncountable rank. Also $S^{\prime}$ is left nonsingular. So $L^{\prime}$, the maximal left ring of quotients of $S^{\prime}$, is such that its left socle is of uncountable rank. This proves that the maximal left rings of quotients of $S$ and $S^{\prime}$ are not isomorphic.

\section{REFERENCES}

[1] G. Abrams, J. Haefner, and A. del Río, The isomorphism problem for incidence rings, Pacific J. Math. 187 (1999), no. 2, 201-214.

[2] F. Al-Thukair, S. Singh, and I. Zaguia, Maximal ring of quotients of an incidence algebra, Arch. Math. (Basel) 80 (2003), no. 4, 358-362.

[3] S. Dăscălescu and L. van Wyk, Do isomorphic structural matrix rings have isomorphic graphs? Proc. Amer. Math. Soc. 124 (1996), no. 5, 1385-1391.

[4] J. Froelich, The isomorphism problem for incidence rings, Illinois J. Math. 29 (1985), no. 1, 142-152.

[5] T. Y. Lam, Lectures on Modules and Rings, Graduate Texts in Mathematics, vol. 189, Springer-Verlag, New York, 1999.

[6] U. Leron and A. Vapne, Polynomial identities of related rings, Israel J. Math. 8 (1970), 127137. 
[7] E. Spiegel, Essential ideals of incidence algebras, J. Austral. Math. Soc. Ser. A 68 (2000), no. 2, 252-260.

[8] E. Spiegel and C. J. O'Donnell, Incidence Algebras, Monographs and Textbooks in Pure and Applied Mathematics, vol. 206, Marcel Dekker, New York, 1997.

[9] R. P. Stanley, Structure of incidence algebras and their automorphism groups, Bull. Amer. Math. Soc. 76 (1970), 1236-1239.

[10] E. R. Voss, On the isomorphism problem for incidence rings, Illinois J. Math. 24 (1980), no. 4, 624-638.

Surjeet Singh: Department of Mathematics, King Saud University, PO Box 2455, Riyadh 11451, Kingdom of Saudi Arabia

E-mail address: ssingh@ksu.edu.sa

Fawzi Al-Thukair: Department of Mathematics, King Saud University, PO Box 2455, Riyadh 11451, Kingdom of Saudi Arabia 


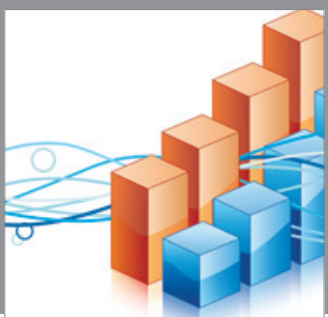

Advances in

Operations Research

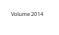

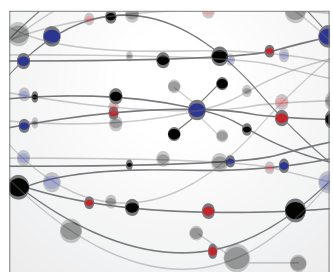

\section{The Scientific} World Journal
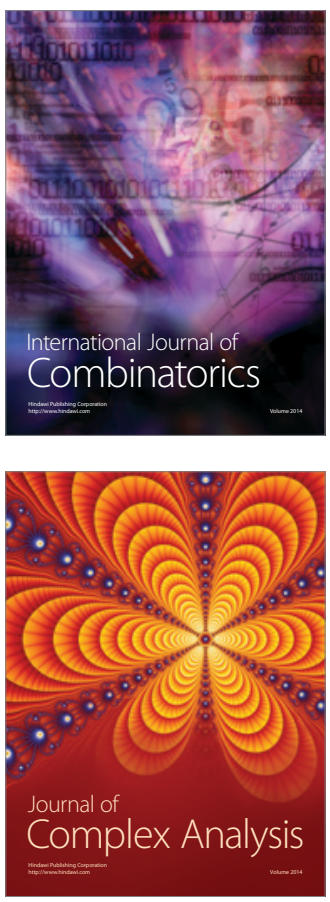

International Journal of

Mathematics and

Mathematical

Sciences
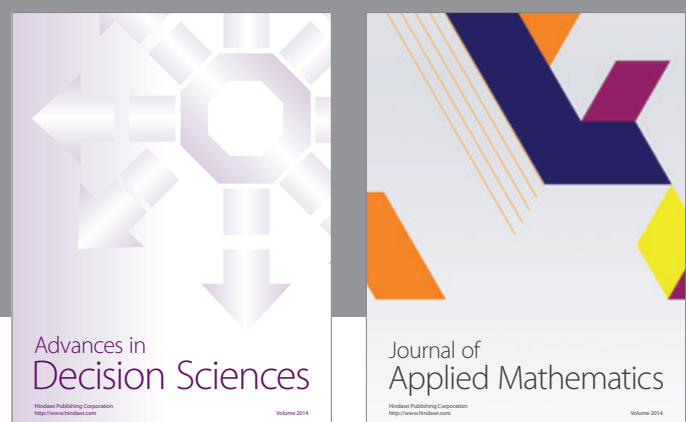

Journal of

Applied Mathematics
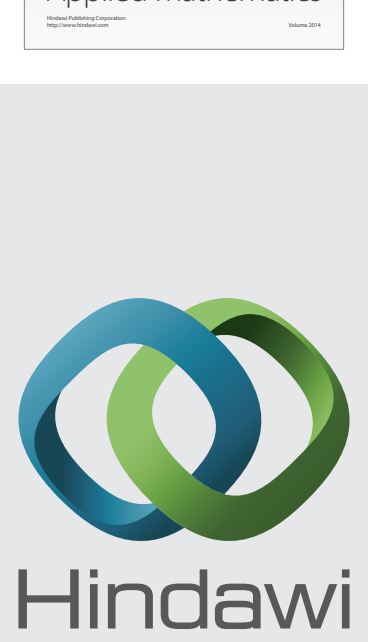

Submit your manuscripts at http://www.hindawi.com
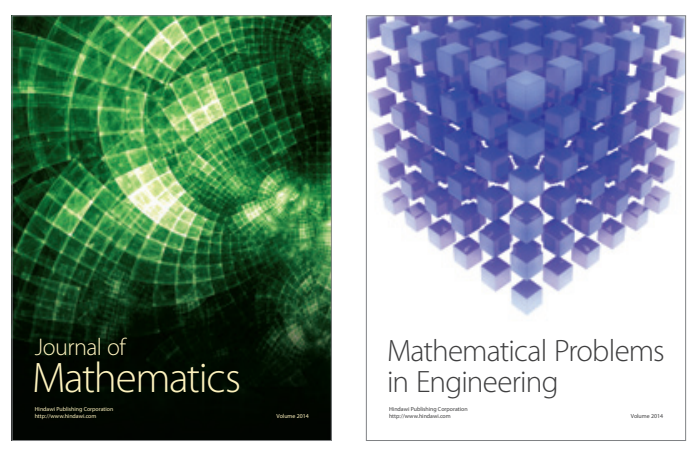

Mathematical Problems in Engineering
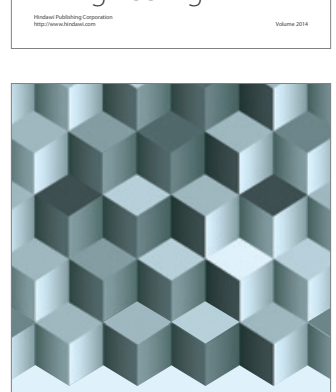

Journal of

Function Spaces
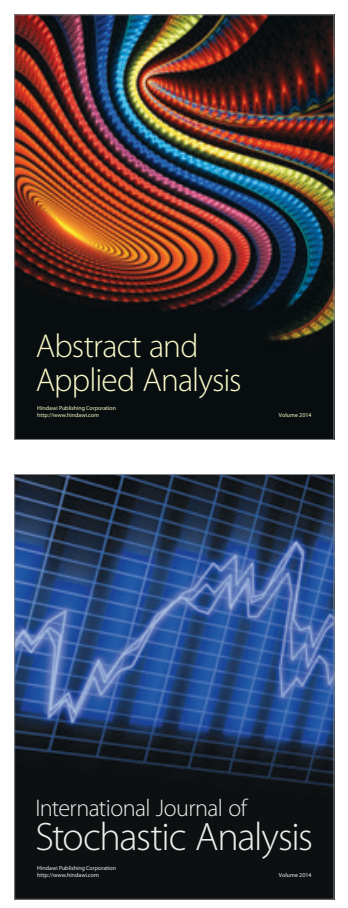

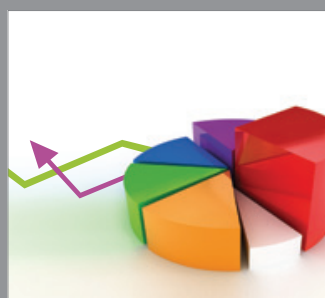

ournal of

Probability and Statistics

Promensencen
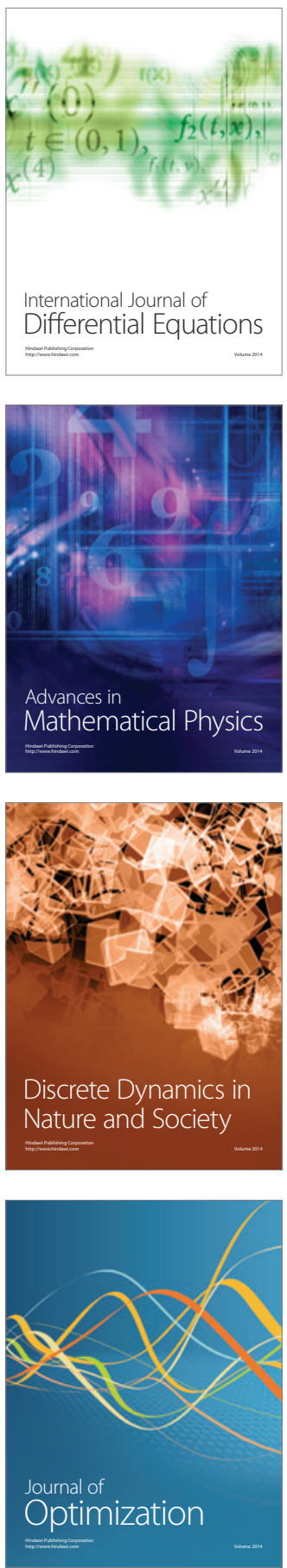